\title{
Envejecimiento y sexualidad femenina
}

\author{
Bárbara Luque Salas \\ Universidad de Córdoba (España)
}

\begin{abstract}
El trabajo que se presenta parte de una investigación más amplia cuyo objetivo principal ha sido profundizar en el conocimiento de la experiencia y la vivencia de la sexualidad en mujeres mayores de 50 años, a partir de su propia voz. Contamos con una muestra de 729 mujeres con edades comprendidas entre los 50 y 80 años. La investigación se enmarca dentro de la metodología cualitativa de investigación feminista. Los datos, cuantitativos y cualitativos, se han recogido a través de un cuestionario de elaboración propia y de entrevistas grupales. Los resultados obtenidos muestran la satisfacción de las mujeres mayores - de todas las edades - con su vida sexual y la importancia de los aspectos contextuales y relacionales en la sexualidad de las mujeres. El autoerotismo aparece como una práctica instaurada en este grupo de mujeres mayores y destacan algunos cambios que desean experimentar en su vida sexual actual en torno al deseo de una sexualidad más sensual y afectiva, con un reclamo de relaciones más apasionadas y frecuentes. Los datos revelan una diferencia cualitativa en la experiencia sexual después de los 70 años.
\end{abstract}

Palabras clave: Envejecimiento, sexualidad, mujeres mayores, investigación feminista.

Aging and women's sexuality. We present results of our research, which has been aimed at understanding the experience, practice, and sexual life in women over 50 years of age. We studied a sample of 729 women of between 50 and 80 years of age. The research is part of qualitative feminist research methodology. Both qualitative and quantitative data have been collected through focus groups and a questionnaire drawn up by our research team. The results show the satisfaction of older women-of all ages-with their sex life and the importance of contextual and relational sexuality issues of women. Autoeroticism is the most established sexual practice in this group of older women and highlights who want to experience some changes in their current sex life around the desire for a more sensual and emotional sexuality, with a claim of more passionate and frequent relations. The data collected reveal a qualitative difference in the reality of sex over the age of 70 .

Keywords: Aging, sexuality, older women, feminist investigation.

Correspondencia: Bárbara Luque Salas. Facultad de Ciencias de la Educación. Universidad de Córdoba. Av. San Alberto Magno, s/n. C.P.: 14071. Córdoba (España). E-mail: bluque@uco.es 
La sexualidad de las mujeres mayores ha sido uno de los temas que más silencios ha ocupado en nuestra sociedad. Las investigaciones realizadas hasta ahora han sido de carácter estadístico, centradas fundamentalmente en las llamadas 'disfunciones sexuales' y realizadas con poblaciones fundamentalmente masculinas. Los importantes cambios demográficos acontecidos en el último siglo, han permitido que un número importante de personas se sitúen en la edad mayor en excelentes condiciones físicas y mentales; una población de hombres y mujeres mayores e incluso muy mayores que puede mantenerse activa sexualmente. Esta realidad social y demográfica choca con numerosas ideas y creencias acerca de la sexualidad en la edad mayor.

A pesar de que en los últimos años ha habido un cambio notable en las actitudes hacia la sexualidad, la creencia social de que las personas mayores son asexuales, que no tienen relaciones ni deseos de carácter sexual, está fuertemente arraigada en nuestra cultura, por lo que no es de extrañar la escasez de estudios que iluminen esta faceta de su vida. La falta de interés por parte de la investigación acerca de este tema, unida a la resistencia de las propias personas mayores a hablar acerca de su vida sexual, han contribuido a la ausencia de una información verdadera y clarificadora, especialmente cuando se trata de mujeres mayores. A este respecto, los primeros estudios acerca de la sexualidad en los que se incluyen mujeres mayores son los llevados a cabo por Masters y Johnson (1966) en los que se constata que la capacidad de goce sexual de las mujeres no decrece con la edad.

Las ideas y prácticas relacionadas con la sexualidad que se sostienen en la juventud se convierten en un sistema de creencias que determina la vivencia satisfactoria de la sexualidad en la edad mayor. Determinados mandatos culturales interfieren de manera clara en la sexualidad de las mujeres mayores: la idea de que sexualidad es fundamentalmente una relación genital o que la sexualidad 'de verdad' es heterosexual; los sentimientos de culpa que rodean el autoerotismo o la idea de que el sexo requiere forzosamente una relación de amor o la vinculación entre sexualidad y maternidad y la asunción cultural de que la feminidad implica pasividad y falta de iniciativa en la relación sexual. Este sistema de creencias conlleva un lastre para la vivencia de la sexualidad en todas las edades, pero de manera especial ha limitado la experiencia erótica de las mujeres que hoy son mayores. En nuestra cultura, la actividad sexual de los hombres dispone de una mayor aceptación y valoración social, produciéndose un doble estándar que ofrece permisividad a los varones para actuar como agentes sexuales, pero desvaloriza y estigmatiza a las mujeres que responden a sus deseos sexuales (Sontag, 1972).

En este momento encontramos dos posiciones argumentativas diferentes para explicar la evolución de la conducta afectivo-sexual de los seres humanos a lo largo de la vida. Por una parte, los planteamientos de la perspectiva biomédica, positivista, que consideran la sexualidad como algo intrínseco, natural y universal, siendo la sexualidad 
de los varones la medida desde la que se realiza cualquier evaluación, diagnóstico y tratamiento. Este planteamiento pasa por alto los aspectos contextuales que pueden afectar la sexualidad. En una línea similar, encontramos los argumentos de la teoría evolucionista de las estrategias sexuales que defienden que el deseo femenino disminuye de manera natural con la edad, a medida que se va aproximando la pérdida de la fertilidad, entendiendo que la reproducción es la base de la sexualidad humana (Buss, 1998). Por otra parte, disponemos de las argumentaciones de la perspectiva interpretativa social que señalan el peso del contexto y de los factores intrapersonales, interpersonales y socioculturales que se ponen en juego en la experiencia sexual de las mujeres de todas las edades, pero especialmente a partir de la mediana edad. Estos planteamientos son similares a las aportaciones críticas de la epistemología feminista y de las perspectivas culturales, que centran su explicación en la comprensión de los elementos contextuales, emocionales y afectivos que influyen sobre las vidas de las mujeres, su experiencia y que determinan las posibilidades de gestión de su propia sexualidad. Estos modelos utilizan metodologías y fuentes de datos diversas, a través de las cuales examinan las experiencias sexuales y el marco cultural que las construye, y llevan a cabo una crítica científica y rigurosa de la investigación biomédica existente acerca del deseo sexual femenino (Freixas y Luque, 2009).

Las dificultades sexuales no son parte intrínseca del envejecer, sino un complejo conjunto determinado por los valores y las expectativas culturales acerca de la sexualidad. De hecho, en algunas mujeres se da un aumento de la actividad sexual, al menos en el caso de quienes legitiman su deseo y lo ponen en práctica, o en el de quienes consiguen transformar su relación con su cuerpo y/o con su pareja; también en el de aquellas que se animan a explorar nuevos caminos (Freixas, Luque y Reina, 2010). La literatura disponible constata el hecho de que los problemas sexuales de las mujeres son multifactoriales y tienen mucho que ver con la educación y con la relación que se mantiene con la pareja o consigo misma (Wood, Mansfield y Kock, 2007).

En cuanto a la vivencia de la sexualidad en el ciclo vital, en función de la opción sexual, la investigación suele partir no sólo de un modelo androcéntrico, sino que parte también del presupuesto de la heterosexualidad normativa, según el cual el sexo 'real' se produce entre mujeres y hombres (Rich, 1980, 2001). Las mujeres lesbianas mayores tienen que afrontar un triple estándar del envejecimiento: a la invisibilidad de ser mujeres y mayores se añade la de ser lesbianas.

\section{MÉTODO}

Presentamos una síntesis abreviada de los resultados de nuestra investigación que ha tenido como objetivo conocer la vivencia, la práctica y la experiencia de la sexualidad en mujeres mayores de 50 años. 


\section{Participantes}

El proceso de búsqueda de participantes se ha realizado a partir de diversas fuentes y en gran medida ha gozado de la colaboración de personas que tienen acceso a grupos de mujeres mayores. Disponemos de 729 cuestionarios válidos de mujeres -reclutadas utilizando la técnica de la "bola de nieve": conocer a algunas informantes y lograr que ellas nos presenten a otras (Taylor y Bogdan, 1984, 1986:109) — que tienen más de cincuenta años y de cualquier opción sexual.

\section{Instrumentos}

La recogida de datos se ha realizado a través de un cuestionario de elaboración propia y de entrevistas grupales que nos han permitido obtener un conocimiento cualitativo y discursivo de los resultados del cuestionario. Para el análisis de los datos se ha llevado a cabo una codificación abierta.

\section{Procedimiento}

Esta investigación se enmarca dentro de la metodología cualitativa de investigación feminista de carácter interpretativo en la que se reconoce la validez de la experiencia de las mujeres a partir de su propia voz (Hesse-Biber y Yaiser, 2004), el papel de la subjetividad en el proceso de investigación (Harding, 1987; Reinharz, 1992), y el carácter político de la investigación y la reflexividad a lo largo de todo el proceso (Crawford y Kimmel, 1999). El método de trabajo ha sido cuantitativo y también cualitativo a partir de las narraciones de las participantes en los diferentes grupos de discusión y en las preguntas abiertas del cuestionario.

\section{RESULTADOS}

La configuración de la población que ha respondido al cuestionario $(N=729)$ se ajusta a los siguientes parámetros:

Tabla 1. Perfil participantes

\begin{tabular}{|c|c|c|c|c|c|c|}
\hline & icipantes & $n$ & $\%$ & Participantes & $n$ & $\%$ \\
\hline \multirow{3}{*}{ Edad } & $50-59$ & 492 & 67.49 & Mantienes relaciones sexuales con: & & \\
\hline & $60-69$ & 190 & 26.06 & Pareja afectiva & 478 & 65.57 \\
\hline & 70 y más & 47 & 6.45 & Conmigo misma & 324 & 44.44 \\
\hline \multirow{2}{*}{ Opción sexual } & Heterosexual & 675 & 92.59 & No marca consigo misma & 155 & 21.26 \\
\hline & Lesbian/ Bisexual & 54 & 7.41 & Voluntariam. abstengo & 69 & 9.47 \\
\hline \multirow{3}{*}{ Pareja afectiva } & $\mathrm{Si}$ & 504 & 69.14 & Otra persona & 63 & 8.64 \\
\hline & No & 214 & 29.36 & Más de una persona & 30 & 4.12 \\
\hline & No contesta & 11 & 1.50 & & & \\
\hline
\end{tabular}

Prácticas sexuales actuales: Tres de las prácticas propuestas en la encuesta superan el 50\% de implantación —-sexo vaginal, besos y caricias y masturbación. Otras 
prácticas frecuentes son 'masturbación mutua' y 'sexo oral'. El sexo anal y el cibersexo son prácticas puntuales.

Tabla 2. Prácticas sexuales

\begin{tabular}{ccccccccc}
\hline & Sexo vaginal & Besos y caricias & Masturbación & Masturbación mutua & Sexo oral & Ninguna & Sexo anal & Cibersexo \\
\hline$f$ & 463 & 402 & 368 & 293 & 275 & 76 & 57 & 14 \\
$\%$ & 63.51 & 55.14 & 50.48 & 40.19 & 37.72 & 10.43 & 7.82 & 1.92 \\
\hline
\end{tabular}

Satisfacción obtenida: En términos globales aparece una satisfacción alta y media-alta con su sexualidad.

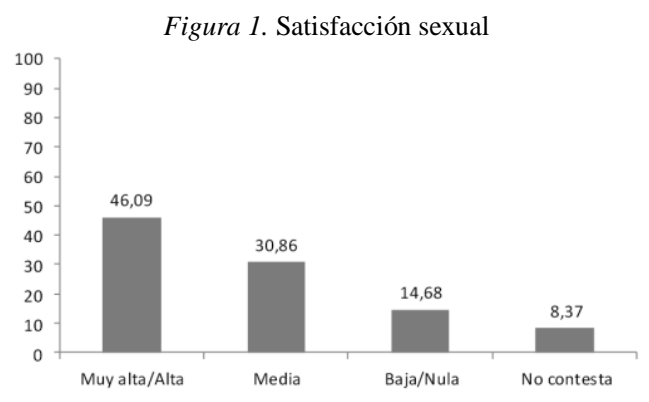

Menos de un $15 \%$ se siente insatisfecha con su sexualidad actual. En los grupos de 50-59 años y 60-69 años la vivencia de la sexualidad es claramente satisfactoria. Este bienestar disminuye a partir de los 70 años, aunque $21 \%$ de las mujeres de más de 70 años confirma que su sexualidad es plenamente satisfactoria y el $19 \%$ obtiene un bienestar medio; por lo que el $40 \%$ se sienten a gusto con su vida sexual en edades superiores a los 70 años. Los niveles de insatisfacción aumentan con la edad, duplicándose de década en década: $10 \%$ de las más jóvenes, el 20\% de las medianas y el $38 \%$ de las más mayores argumentan insatisfacción.

Deseos de cambio o mejora: Cuando reflexionan acerca de qué desearían cambiar o mejorar en su vida sexual actual vemos que las demandas se pueden organizar de acuerdo con las siguientes unidades de significado:

Tabla 3 Cambios deseados

\begin{tabular}{|c|c|c|c|c|c|c|c|c|c|c|}
\hline \multicolumn{5}{|c|}{ Prolegómenos y desarrollo de calidad } & \multicolumn{2}{|c|}{$\begin{array}{c}\text { Mayor intensidad } \\
\text { sexual }\end{array}$} & \multicolumn{2}{|c|}{$\begin{array}{c}\text { Mayor libertad } \\
\text { personal }\end{array}$} & \multicolumn{2}{|c|}{$\begin{array}{c}\text { En relación a la } \\
\text { pareja }\end{array}$} \\
\hline $\begin{array}{c}\text { Más } \\
\text { sensualidad }\end{array}$ & $\begin{array}{c}\text { Más } \\
\text { caricias }\end{array}$ & $\begin{array}{c}\text { Más } \\
\text { ternura }\end{array}$ & $\begin{array}{c}\text { Más } \\
\text { tiempo }\end{array}$ & $\begin{array}{c}\text { Más } \\
\text { intimidad }\end{array}$ & $\begin{array}{c}\text { Más } \\
\text { pasión }\end{array}$ & $\begin{array}{c}\text { Más } \\
\text { frecuencia }\end{array}$ & $\begin{array}{c}\text { Menos } \\
\text { obligación }\end{array}$ & $\begin{array}{l}\text { Más } \\
\text { iniciat }\end{array}$ & $\begin{array}{l}\text { Más } \\
\text { respeto }\end{array}$ & $\begin{array}{l}\text { Menos } \\
\text { frecuencia }\end{array}$ \\
\hline 31.28 & 29.22 & 23.18 & 18.93 & 9.33 & 29.22 & 29.99 & 17.42 & 14.13 & 4,12 & 4,94 \\
\hline \multicolumn{5}{|c|}{816 respuestas } & \multicolumn{2}{|c|}{431 respuestas } & \multicolumn{2}{|c|}{230 respuestas } & \multicolumn{2}{|c|}{66 respuestas } \\
\hline
\end{tabular}

Las demandas más frecuentes son, por una parte el deseo de una sexualidad más delicada y dedicada, más tierna y sensual. Una sexualidad claramente más sensual, más pausada en la que la calidad de la relación prevalezca por encima de la sexualidad 
más estrictamente clásica. Y por otra parte se reclama una relación sexual más apasionada y frecuente. Hay una clara demanda de mayor frecuencia y pasión en las relaciones sexuales. Un buen número de mujeres en la edad mayor desea disfrutar de más sexo del que tiene. El mayor deseo de pasión y frecuencia sexual predomina en todas las edades, pero es un deseo bastante mayor en las mujeres de opción lesbiana y bisexual (43\%-29\%), mientras que el reclamo afectivo en las mujeres de opción heterosexual es mayor que en las de opción lesbiana/bisexual (21\%-12\%).

En un tercer nivel se sitúan las reflexiones críticas acerca de la gestión de su propia sexualidad. Se reconoce la sumisión a los mandatos socioculturales que inducen a hacer sexo con el fin de contentar a la pareja. En esta situación encontramos al 17\% de las participantes, porcentaje que muestra el peso de la educación restrictiva de nuestra sociedad. Por otra parte se plantea la necesidad de disponer de una actitud personal más activa especialmente en cuanto a tomar la iniciativa en las relaciones sexuales se refiere $(14 \%)$.

Evolución del deseo sexual: Uno de los debates clave en torno a la sexualidad de las mujeres en el periodo postmenopáusico gira en torno a la pérdida de deseo sexual, argumento sobre el que pivota gran parte de las explicaciones biologicistas y evolucionistas. Las respuestas de nuestras participantes acerca del tema muestran los siguientes porcentajes:

Tabla 4. Evolución del deseo sexual

\begin{tabular}{lrrrrrrrrrc}
\hline & \multicolumn{2}{c}{ Ha disminuido } & \multicolumn{2}{c}{ Ha aumentado } & \multicolumn{2}{c}{ No ha cambiado } & \multicolumn{2}{c}{ No contesta } & \multicolumn{2}{c}{ Total } \\
\cline { 2 - 14 } & \multicolumn{1}{c}{$n$} & \multicolumn{1}{c}{$n$} & \multicolumn{1}{c}{$\%$} & \multicolumn{1}{c}{$\%$} & $\%$ & $n$ & $\%$ & $n$ & $\%$ \\
\hline 50-59 años & 328 & 66.67 & 101 & 20.53 & 8 & 1.63 & 55 & 11.18 & 492 & 100 \\
\hline 60-69 años & 135 & 71.05 & 27 & 14.21 & 4 & 2.11 & 24 & 12.63 & 190 & 100 \\
\hline 70 y más & 36 & 76.6 & 1 & 2.13 & 2 & 4.26 & 8 & 17.02 & 47 & 100 \\
\hline Heterosexual & 466 & 69.04 & 115 & 17.04 & 13 & 1.93 & 81 & 12 & 675 & 100 \\
\hline Lesbian/Bisexual & 33 & 61.11 & 14 & 25.93 & 1 & 1.85 & 6 & 11.11 & 54 & 100 \\
\hline Total & 499 & 68.45 & 129 & 17.7 & 14 & 1.92 & 87 & 11.93 & 729 & 100 \\
\hline
\end{tabular}

Un porcentaje importante de participantes afirma que su deseo sexual ha disminuido en los últimos años; un porcentaje bastante menor, pero no despreciable, piensa que, por el contrario, ha aumentado.

Se observa un descenso progresivo del deseo en función de la edad y también una disminución del número de personas que afirman que su deseo ha aumentado con los años, siendo casi nulo en el caso de las mujeres a partir de los 70 años. En el grupo de las mujeres más jóvenes los dos tercios afirman que su deseo ha perdido intensidad, aunque destacamos que una quinta parte de ellas constata que su deseo ha mejorado con los años. Este es un dato de interés. En cuanto a la opción sexual el sostenimiento del deseo se muestra mayor en las mujeres lesbianas y bisexuales, puesto que su deseo ha disminuido en una proporción algo menor que en el caso de las mujeres heterosexuales 
y, a su vez, un porcentaje algo mayor de ellas afirma que su deseo en los últimos años ha aumentado.

Las prácticas de autoerotismo: A esta pregunta responden casi todas las participantes. Excluyendo a quienes no responden y quienes afirman que nunca se han masturbado, constatamos que el $80 \%$ responde a esta cuestión aportando diversas y múltiples perspectivas. Hemos agrupado las respuestas que la encuesta ofrece en dos bloques: a) sobre la instauración de la práctica de la masturbación a lo largo del tiempo y b) sobre la frecuencia con que se lleva a cabo en la actualidad.

La práctica del autoerotismo a lo largo del tiempo: Informaciones referentes a la temporalidad de la práctica de la masturbación, que va desde quienes nunca se han masturbado, hasta quienes se iniciaron de pequeñas, en un arco que iría desde la precocidad (14\% afirma que empezaron antes de los 10 años), a la 'tardanza', que oscila entre quienes en tiempos más jóvenes nunca lo hicieron, pero sí lo hacen ahora o las que no lo han hecho nunca. El porcentaje de mujeres que afirman que nunca se han masturbado aumenta de manera muy clara a partir de los 70 años. En cuanto a la opción sexual se puede observar en algunos aspectos una mayor actividad en cuanto al autoerotismo por parte de las mujeres lesbianas y bisexuales.

Frecuencia reconocida: El 60\% - 437 participantes- se masturba, con frecuencia o esporádicamente. Un porcentaje de gran calado que nos permite destacar esta práctica como un elemento de interés y satisfacción en todas las edades de la vida, que se mantiene a lo largo de los años. En estos apartados también encontramos porcentajes ligeramente mayores de actividad autoerótica en las mujeres de opción lesbiana y bisexual.

Gustos y deseos en relación a la sexualidad actual: Tres preguntas abiertas del cuestionario tratan sobre aspectos de la vivencia de la sexualidad, indagan acerca de lo que 'gusta', lo que 'se desea' o 'gustaría probar'. Las informaciones obtenidas indican el valor contextual que la sexualidad tiene en la vida de las mujeres. De las numerosas respuestas sólo un pequeño grupo enumera/propone actividades y prácticas directamente sexuales, mientras que el peso de las respuestas se centran en elementos afectivos y relacionales. Nos llama la atención el hecho de que en una población de mujeres mayores se den tantas concreciones de placeres sexuales. Es decir, hay deseo y se concreta con precisión. Encontramos un buen número de afirmaciones que valoran la gestión de la propia sexualidad: los sentimientos de libertad, de no dependencia, la capacidad de iniciativa, el conocimiento del propio cuerpo y del deseo, que nos indican que las mujeres de todas las edades van tomando las riendas de su propia sexualidad y que ésta no queda a expensas de tener o no pareja, o de las oportunidades que la sociedad les asigna. La salud propia y de la pareja se constituyen en previos imprescindibles.

Ideas y creencias en torno a la sexualidad en la edad mayor: Dos preguntas del cuestionario se centran en las ideas y creencias acerca de la sexualidad. A través de 
ellas hemos pretendido profundizar en los elementos internos que rigen y valoran la sexualidad propia y qué dificultades se suponen predominantes en las mujeres mayores para la vivencia de una sexualidad satisfactoria. La 'falta de deseo' es considerada por más de la mitad de las participantes como la dificultad más importante con que se enfrentan las mujeres mayores. A continuación, encontramos tres circunstancias a las que se refiere una tercera parte de todas las participantes: 1) falta de pareja; 2) cansancio/estrés y 3) falta de excitación. Este último se relaciona muy directamente con la falta de deseo que ha sido la respuesta más unánime.

Si bien la falta de deseo es, con diferencia, la explicación que ofrece más de la mitad de las mujeres heterosexuales, en el caso de las mujeres lesbianas y bisexuales este argumento desciende a un cuarto lugar. Para este grupo de población la afirmación más contundente acerca de las dificultades con que se encuentran las mujeres de edad en su vida sexual radica en la falta de pareja, seguida del cansancio y estrés y de las dificultades con la imagen y autoestima corporales. El hecho de 'no tener pareja' es una realidad que denuncian de manera homogénea nuestras participantes de todas las edades, con porcentajes superiores al $30 \%$ todas ellas.

\section{CONCLUSIONES}

La sexualidad de las mujeres en la edad mayor es una experiencia múltiple y diversa, que tiene mucho que ver con la historia personal; no hay un discurso único que pueda resumir y explicar la vida afectivo/sexual de las mujeres al envejecer. Destacamos algunas tendencias observadas en los resultados obtenidos, partiendo de la idea de que el carácter multidimensional de la sexualidad hace imposible encerrar en pocas palabras la complejidad de la sexualidad en las mujeres después de la mediana edad.

El silencio en torno a la sexualidad y la dificultad para hablar de ella constituyen uno de los obstáculos para la normalización de la sexualidad de las mujeres en la edad mayor. Cuando les hemos preguntado por su sexualidad, reconocen una alta implicación en la actividad sexual, cuando las circunstancias básicas de disposición de una pareja y de un nivel de salud suficiente lo permiten. También hemos observado, al estudiar los datos por edades, que hay un salto cualitativo en la experiencia y realidad sexual a partir de los 70 años, lo cual no significa que el deseo y la emoción sexual desaparezcan con la edad.

En relación a la satisfacción con su sexualidad los datos muestran un alto nivel de satisfacción global de las mujeres mayores con su vida sexual actual, lo que contradice la creencia popular acerca de la asexualidad de las mujeres mayores. El nivel de satisfacción es ligeramente superior en las participantes lesbianas y bisexuales.

Los datos, en cuanto a los cambios que se desearía introducir en la vida sexual destacan el deseo de unas relaciones de mayor calidad afectiva (más sensualidad, más 
caricias, más ternura), que confirma el valor contextual y afectivo que las mujeres de todas las edades otorgan a la sexualidad, así como el deseo de una mayor intensidad y frecuencia en las prácticas sexuales actuales.

En resumen, podríamos sintetizar estos datos destacando la implicación de las mujeres de todas las edades en la actividad sexual, cuando las circunstancias básicas de disposición de una pareja y de un nivel de salud suficiente lo permiten, destacando el autoerotismo como una práctica sexual instaurada y mantenida.

\section{Agradecimientos}

Esta investigación ha dispuesto de la financiación del Ministerio de Ciencia e Innovación, a través de una acción complementaria del Plan Nacional de I+D+I CSO2008-04358-E.

\section{REFERENCIAS}

Buss, D.M. (1998). Sexual Strategies Theory: Historical Origins and Current Status. The Journal of Sex Research, 35(1), 19-31.

Crawford, M. y Kimmel, E. (1999). Promoting Methodological Diversity in Feminist Research. Psychology of Women Quarterly, 23(1), 1-6.

Freixas, A. y Luque, B. (2009). El secreto mejor guardado: la sexualidad de las mujeres mayores. Politica y Sociedad, 46(1-2), 191-203.

Freixas, A., Luque, B. y Reina, A. (2010). Secretos y silencios en torno a la sexualidad de las mujeres mayores. Debate Feminista, 42, 35-51.

Harding, S. (1987). Feminism and Methodology: Social science issues. Bloomington: Indiana University Press.

Hesse-Biber, S.N. y Yaiser, M.L. (2004). Feminist Perspectives on Social Research. New York: Oxford University Press.

Masters, W.H. y Johnson, V.E. (1966). Human sexual response. Boston: Little Brown.

Rich, A. (1980/ 2001). Heterosexualidad obligatoria y existencia lesbiana. En A. Rich (Ed.), Sangre, pan y poesía. Prosa escogida 1979-1985 (pp. 41-86). Barcelona: Icaria.

Reinharz, S. (1992). Feminist Methods in Social Research. Oxford: Oxford University Press.

Sontag, S. (1972). The double standard of aging. Saturday Review, 29-38.

Taylor, S.J. y Bogdan, R. (1984/1986). Introducción a los métodos cualitativos de investigación. La búsqueda de significados. Buenos Aires: Paidós.

Wood, J.M., Mansfield, P.K. y Kock, P.B. (2007). Negotiating Sexual Agency: Postmenopausal Women's Meaning and Experience of Sexual Desire. Qualitative Health Research, $17(2), 189-200$.

Recibido: 30 de abril de 2014

Recepción Modificaciones: 26 de mayo de 2014

Aceptado: 7 de agosto de 2014 